\title{
T019
}

\section{Seismic Characterisation for Geothermal Energy} Prospecting

\author{
A. Huck* (dGB Earth Sciences), P. de Groot (dGB Earth Sciences), E. \\ Simmelink (TNO), V.P. Vandeweijer (TNO) \& A. Willemsen (IF Technology)
}

The city of The Hague intends to use geothermal energy to heat approx. 4000 houses in a planned urban development area called The Hague South-West. This paper describes the application of advanced seismic interpretation workflows to help positioning a geothermal doublet consisting of one injector - and one producer well. An existing 3D seismic data volume was reprocessed and inverted to acoustic impedance.

The main objectives of the study were to predict the thickness of the target Rijswijk and Delft sandstones and to visualize faults and fractures that might act as permeability barriers between injector and producer.

The inverted acoustic impedance volume revealed several layers of low and high impedances in and around the targeted sandstones in which lateral stratigraphic variations are observed.

To visualize faults and fractures the seismic data were first filtered using two dip-steered filters. Next a similarity volume and a neural network generated Fault Cube were produced and highlighted major and minor fault systems at target levels.

The study results were used to assist in picking the optimal locations for the doublet. The lack of impedance contrasts and available well data prevented further quantitative work like predicting thickness and porosity of the target sandstones. 


\section{Amsterdam 'o9}

\section{Introduction}

This paper describes the results of an advanced seismic interpretation study focusing on a small area located inside the city of The Hague. This work was part of the The Hague South West geothermal project. This high-profile project aims at providing central heating for approx. 4000 houses to be built in the city of The Hague. The project was carried out by a consortium of energy and housing construction companies and covers most of the tasks required for geothermal prospecting (Wolf et al., 2008).

The objective was the extraction of relevant information from the seismic data to help positioning one injector and one producer well. TNO provided the 3D seismic data and an interpreted horizon. IF Technology is the engineering firm assigned to develop the subsurface facilities.

Specific objectives were the inversion of the seismic data to Acoustic Impedance. This inverted volume was used to better localize the top and bottom of the sandstone layers. A second independent objective was the application of filters and computation of attributes for an optimal visualization of faults at the target interval. The interpretation described here allowed the detailed design of two wells, which are planned to be drilled in the Delft sandstone in 2009.

\section{Methodology}

The standard seismic interpretation workflow used for the characterization of hydrocarbon reservoirs needed to be adjusted to meet the different requirements for successful geothermal production. Successful geothermal doublets should be located in a permeable high temperature formation. Therefore sandstone synclines are good potential targets, whereas oil and gas wells are often drilled in anticline structures. Temperatures above 70 degrees are the minimum requirement for heating purposes. Therefore the project was divided into two parts: A reservoir characterisation using acoustic impedance inversion to map the sandstone formation of sufficient depth, and a fault analysis to locate faults that could act as a permeability barrier. Figure 1 shows a basemap of the survey area with the existing wells drilled by the oil and gas industry.

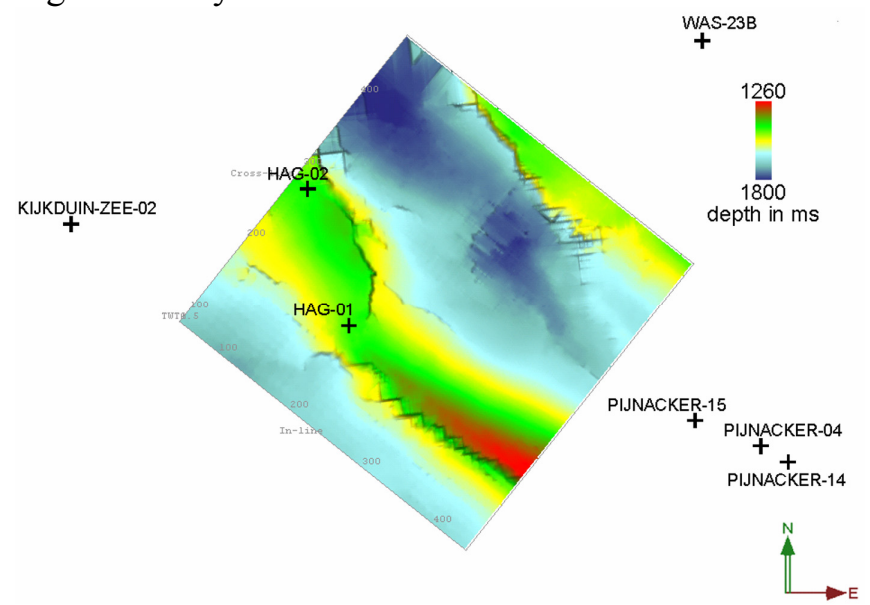

Figure 1: Survey basemap with well positions and base Cretaceous interpretation. The syncline structure is displayed in dark blue.

\section{Acoustic Impedance inversion}

A model-based acoustic impedance inversion was carried out to invert the seismic data to impedance. The initial low frequency model was driven by a single horizon, the base Cretaceous horizon. A statistical wavelet was extracted from the seismic data within the syncline. The main difficulty is this project was the lack of well data. No sonic or density logs 


\section{Amsterdam 'og}

were available for either HAG-01 or HAG-02 wells, Therefore the model had to be built from wells outside the survey area. Checkshots were available for wells outside the survey area and the base Cretaceous horizon was extrapolated outside of the survey to honour those checkshots. For that reason no deterministic wavelet could be computed and the inversion was run using the statistical wavelet. Figure 2 shows the seismic and inverted impedance data along a west-east profile, with the base Cretaceous horizon.
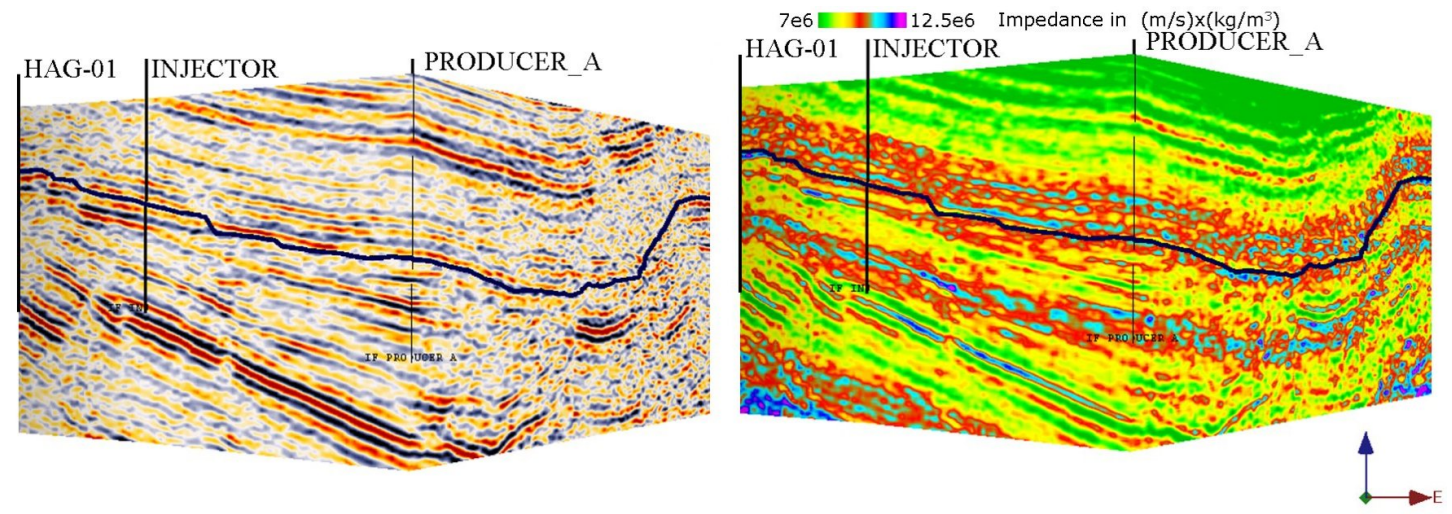

Figure 2: Seismic and inverted impedance profiles in a profile between the existing well $H A G-01$, and the injector and final producer locations .The base Cretaceous horizon is displayed as a blue line.

The main target, the Delft sandstone, is located under the base Cretaceous horizon, below the Rodenrijs claystone. A secondary target, the Rijswijk sandstone, is at the base of the Cretaceous interval.

\section{Fault detection}

Attributes were used in the project to obtain information on the location of faults. Fault detection is in general very complex, as the seismic character of the faults and their surroundings is continuously changing. Faults are generally no single fault planes but complex faulted zones.

Fault enhancement filtering was applied to the seismic data prior to similarity computations to increase the quality of the similarity-based attributes. This post-stack filtering technique increases the contrast and the resolution of the derived similarity volume. It is a structurally oriented filter (using steering) which adapts its filtering characteristics depending on the local character of the seismic. The filter will smooth the seismic away from the faults, and will sharpen the discontinuities at the locations of the faults.

Similarity is the main attribute used for fault detection. This form of coherency expresses how much two or more trace segments look alike. Several time gates were used to discriminate the low scale faults (polygonal, fractures), from high scale faults. Similarity is a non-unique attribute: It will not only show fault related anomalies. A ridge-enhancement filtering metaattribute (Aminzadeh et al, 2005) and a neural network FaultCube (Aminzadeh et al, 2004) were computed from the previously computed similarity.

The ridge-enhancement filter is a method to enhance lineaments related to small-scale faults and fractures. It is a combination of similarity calculations and logic used to sharpen fault images. Application of the ridge-enhancement filtering was needed to enhance small scale faults in the Delft and Rijswijk formations, in particular in the north-eastern part of the survey where multiple low scale faults, fractures and polygonal faulting are visible (Figure 3). 

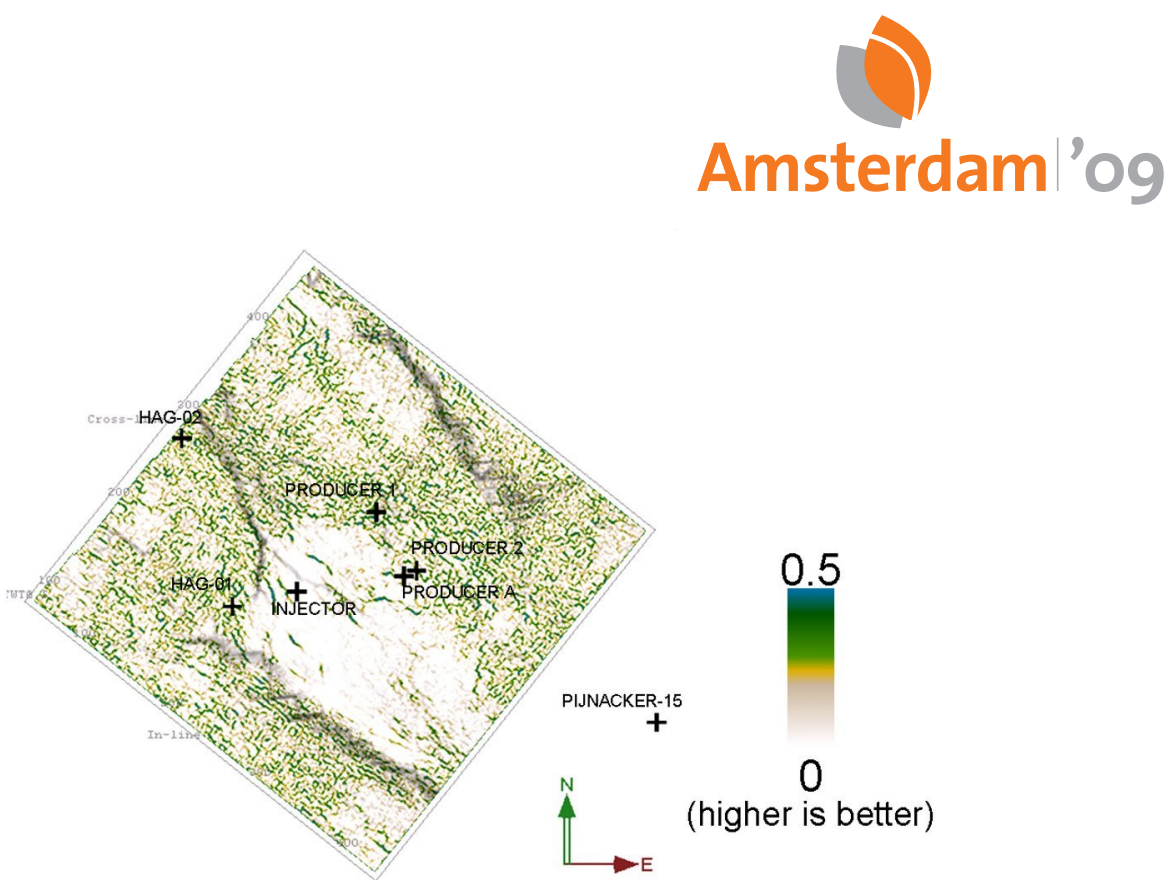

Figure 3: Similarity from ridge-enhancement filter within the Delft sandstone (100ms below base Cretaceous) with the position of the injector and producer wells. The 'Producer A" position was the final position retained.

The fault detection was further extended using a supervised learning approach. The technique consists of a fully connected Multi-Layered-Perceptron (MLP) neural network that learns to classify seismic information into fault probability. The supervised approach emphasizes fault continuity and reduces noisy zones that are not related to faulting. The output FaultCube exhibited clearly the major faults, but for the very faulted north-eastern part the ridgeenhancement volume was preferred.

\section{Integration}

During the course of the study several producer positions were selected by the client based on the results of the inversion and the fault detection and the injector also moved and is now slightly deviated, whereas the top of the injector location was static, being the proposed surface location. The producer locations were tested during the project considering lithology changes, thickness changes and the presence of faults, using both the inverted impedance and ridge-enhancement filter results.

The first two positions were rejected because as they were too close to major faults. The section through the injector and the third producer location did not show any majors faults but only small local discontinuities.

Several clear impedance contrasts were visible on the absolute impedance section but a calibration to the well KDZ-02 showed a poor impedance contrast between sand and claystones. The thickness of first low impedance area below the base Cretaceous was estimated to be $240 \mathrm{~m}$. Wells HAG-01 and HAG-02 encountered a Delft sandstone thickness of respectively 102 and $40 \mathrm{~m}$, therefore the interpretation (Figure 4) suggested that the low impedance area does not correspond to only the Delft sandstone but also incorporates part of the overlaying Rodenrijs and Alblasserdam shales. 


\section{Amsterdam 'o9}

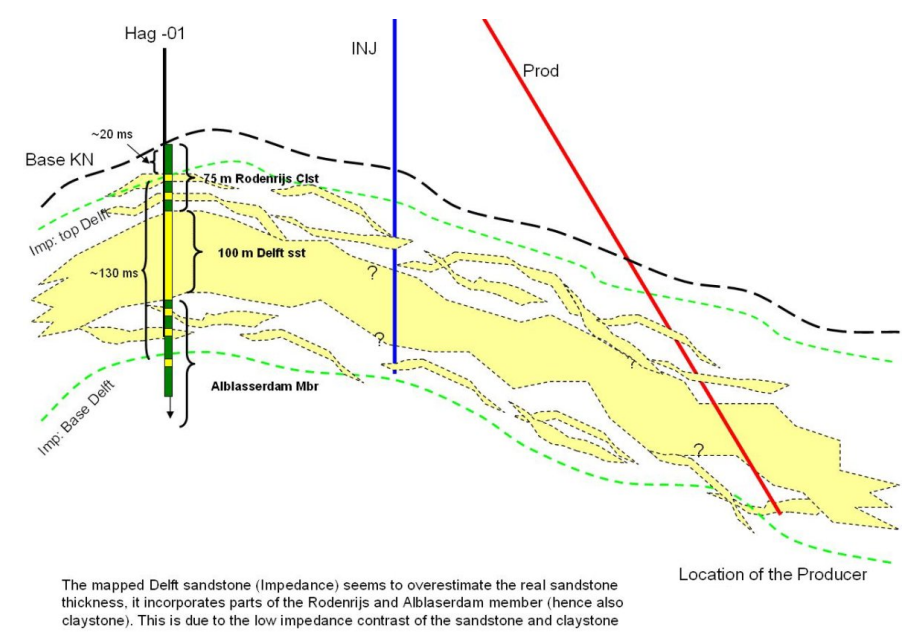

Figure 4: Integration of the well data with the seismic interpretation and impedance inversion results.

\section{Conclusions}

The fault outputs showed major faults that could act as permeability barriers and small-scale faults that do not represent a problem. Based on this information the producer position was moved several times. The final section showed only small scale discontinuities.

The Impedance inversion delivered a volume where lateral stratigraphic changes can be observed between the wells. The volume exhibits an alternation of low and high impedance layers. The integration of the inversion results to the well data showed that impedance contrasts could not be used directly to map the top and base of the Rijswijk and Delft sandstones. As a result no reliable thickness estimation could be provided.

The well doublet will be drilled in 2009 .

\section{Acknowledgements}

We thank Aardwarmte Den Haag VOF for the authorization to present this work.

\section{References}

Aminzadeh, F., de Groot, P., 2005, A neural networks based seismic object detection technique, SEG technical program expanded abstracts, pp 775-778.

Aminzadeh, F., de Groot, P., 2004, Soft Computing for qualitative and quantitative seismic object and reservoir property prediction, Part 1, Neural network applications, First Break, Volume 22, pp 49-54.

Wolf, K-H.A.A., Willemsen, A., Bakker, T.W., Wever, A.K.T., Gilding, D.T., 2008, 70th EAGE Conference \& Exhibition, extended abstracts, D021. 